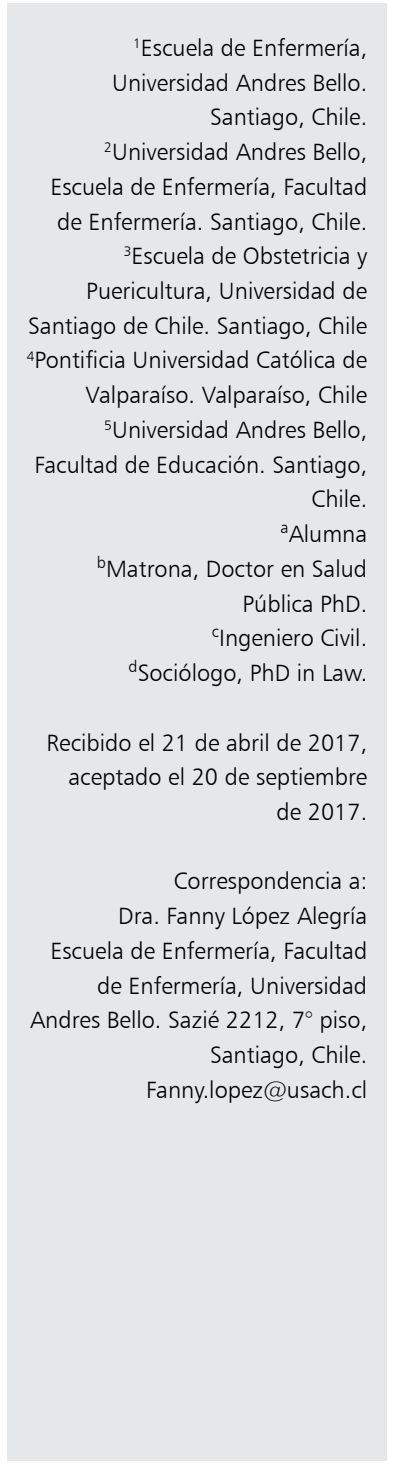

\section{Calidad de sueño y rendimiento académico en alumnos de educación secundaria}

\author{
MAITHE BUGUEÑO ${ }^{1, \mathrm{a}}$, CAROLINA CURIHUAL $^{1, \mathrm{a}}$, \\ PAULINA OLIVARES ${ }^{1, \mathrm{a}}$, JOSEFA WALLACE $^{1, \mathrm{a}}$, FANNY LÓPEZ-ALEGRÍA ${ }^{2,3, \mathrm{~b}}$, \\ GONZALO RIVERA-LÓPEZ ${ }^{4, c}$, JUAN CARLOS OYANEDEL ${ }^{5, \mathrm{~d}}$
}

\section{Quality of sleep and academic performance in high school students}

Background: Sleeping and studying are the day-to-day activities of a teenager attending school. Aim: To determine the quality of sleep and its relationship to the academic performance among students attending morning and afternoon shifts in a public high school. Material and Methods: Students of the first and second year of high school answered an interview about socio-demographic background, academic performance, student activities and subjective sleep quality; they were evaluated using the Pittsburgh Sleep Quality Index (PSQI). Results: The interview was answered by 322 first year students aged $15 \pm 5$ years attending the morning shift and 364 second year students, aged $16 \pm 0.5$ years, attending the afternoon shift. The components: sleep latency, habitual sleep efficiency, sleep disturbance, drug use and daytime dysfunction were similar and classified as good in both school shifts. The components subjective sleep quality and duration of sleep had higher scores among students of the morning shift. The mean grades during the first semester of the students attending morning and afternoon shifts were 5.9 and 5.8, respectively (of a scale from 1 to 7). Among students of both shifts, the PSQI scale was associated inversely and significantly with academic performance. Conclusions: A bad sleep quality influences academic performance in these students.

(Rev Med Chile 2017; 145: 1106-1114)

Key words: Adolescent; Sleep; Students.
$\mathrm{E}$ 1 sueño es una función biológica fundamental y es durante este proceso que el cerebro descansa y se recarga para estar activo durante el día, lo que es importante en la etapa de la adolescencia para desarrollar las actividades escolares y por consiguiente obtener un óptimo rendimiento académico ${ }^{1-3}$. El concepto "calidad del sueño" es un constructo que puede ser evaluado mediante escalas como el "índice de calidad de sueño de Pittsburgh" (PSQI) ${ }^{4,5}$.

En el año 2010, la Encuesta Nacional de Salud mostró que $63,2 \%$ de los chilenos manifiesta problemas para dormir, siendo los jóvenes la población más afectada con una prevalencia de $64,8 \%$, evidenciándose esta situación como un problema de salud pública ${ }^{6}$. Gran parte de esta población, está cursando su etapa estudiantil en diversos establecimientos educacionales. Uno de estos establecimientos es el Instituto Nacional José Miguel Carrera, que es la institución educacional pública más antigua de Chile, fundada en el año 1813. Esta institución posee un alto prestigio y 
es considerada la cuna de la educación chilena, ya que posee la tradición de seleccionar y formar estudiantes de alto rendimiento académico, lo que se traduce en altos resultados en las Pruebas de Selección (PSU), para el ingreso al Sistema Universitario del país?

Este articulo pretende evaluar la calidad subjetiva del sueño y su relación con el rendimiento académico de los estudiantes de la jornada de la mañana $\left(2^{\circ}\right.$ año de enseñanza media) y de la jornada de la tarde ( $1^{\circ}$ año de enseñanza media) del Instituto Nacional José Miguel Carrera, Santiago, año lectivo 2016.

\section{Materiales y Métodos}

El tipo de estudio fue observacional, descriptivo, cuantitativo, transversal y de carácter analítico. El universo de estudio correspondió a los estudiantes que están cursando $1^{\circ}$ y $2^{\circ}$ año de enseñanza media del Instituto Nacional José Miguel Carrera, matriculados durante el año 2016, lo que corresponde a un total de 1.440 alumnos. La muestra de estudio fue calculada asumiendo varianza máxima, con un margen de error de 3,5\% para un nivel de confianza de $99 \%$, obteniéndose una muestra teórica de 686 alumnos a encuestar en el mes de octubre. El criterio de inclusión fue estar cursando $1^{\circ} \mathrm{o} 2^{\circ}$ año de enseñanza media durante el período de recolección de datos. El criterio de exclusión fue estar ausente o con licencia médica en los días de recolección de datos. La muestra total del estudio fue de 686 casos.

Las variables de estudio fueron: antecedentes sociodemográficos, calidad subjetiva del sueño, rendimiento académico y actividades estudiantiles.

El instrumento consta de un cuestionario cuya primera parte corresponde a información sociodemográfica, con variables como edad y comuna de residencia.

En la segunda parte, se incluye la encuesta de Pittsburg de Calidad de sueño (Pittsburgh Sleep Quality Index, PSQI) en su versión en español ${ }^{4,8}$.

En la tercera parte, se incluyeron preguntas relativas a las actividades estudiantiles como: escolaridad, promedio de notas del total de asignaturas del primer semestre lectivo y jornada de clases. Todas las preguntas son relativas a las actividades diarias, excluyendo los días feriados y fines de semana.
Se solicitó el consentimiento informado en forma escrito. El estudio fue autorizado por las autoridades del establecimiento educacional y aprobado por el Comité de Ética de la Escuela de Enfermería de la Universidad Andrés Bello.

Se describieron los componentes del PSQI con la puntuación parcial de cada uno que va de 0 a 3 , es decir un puntaje $0=$ bastante bueno; $1=$ bueno; $2=$ malo y $3=$ bastante malo.

El puntaje global de la PSQI es la suma de los siete componentes, que oscila entre 0 a 21 puntos (mayor puntaje es equivalente a mayor severidad de la mala calidad de sueño). Los participantes con una puntuación global de $<5$ fueron clasificados como buenos dormidores o "sin problemas de sueño" y los $\geq 5$ fueron clasificados como malos dormidores. Las notas promedio del total de asignaturas del semestre son clasificadas de acuerdo al sistema de educación media en Chile ${ }^{9,10}$.

En los análisis se utilizó el Statistical Package for the Social Sciences (SPSS) versión 21. Los datos fueron analizados mediante estadística descriptiva y análisis correlacionales, de varianza y regresión logística binaria.

\section{Resultados}

La muestra de estudio está constituida por 686 alumnos, divididos en 322 alumnos (46,9\%) que cursan $1^{\circ}$ año de enseñanza media (jornada de la tarde) y 364 alumnos $(53,1 \%)$ que cursan $2^{\circ}$ año de enseñanza media (jornada de la mañana). La edad promedio de los alumnos de $1^{\circ}$ año es de 14,7 años $(\mathrm{DE}=0,56)$ y de los de $2^{\circ}$ año es de 15,6 años $(\mathrm{DE}=0,54)$. Los lugares de habitación de los alumnos se distribuyen en la mayoría de las comunas de Santiago, teniendo una mayor concentración en las comunas de Maipú con 81 estudiantes $(11,8 \%)$ y Santiago Centro con 70 estudiantes $(10,2 \%)$, esta última es la comuna de ubicación del Instituto.

Las clases de la jornada de la mañana se inician a las 7:45 y de la tarde a las 14:05. Así, los alumnos de la jornada de la mañana, en su mayoría 248 $(68,1 \%)$ se levantan entre las 6:00-6:59 h y los de la tarde, 197 estudiantes $(61,3 \%)$ entre las 8:00 y las 9:59 h (Tabla 1).

A partir de las 21:00 h los alumnos se van a dormir, siendo el horario más frecuente para los alumnos de la jornada de la mañana, entre las 
23:00-23:59 y de las 01:00-01:59 para los de la tarde (Tabla 2).

En relación a los componentes del Índice Pittsburg de Calidad de Sueño, tenemos que el $1^{\circ}$ componente, calidad subjetiva de sueño, para la mayoría de los alumnos, $220(58,9 \%)$ de la jornada de la mañana es clasificada como buena y aún mejor para los alumnos de la jornada de la tarde $235(72,1 \%)$ que es calificada de bastante buena (Figura 1).

$\mathrm{El} 2^{\circ}$ componente, latencia de sueño, la mayoría de los alumnos $282(75,6 \%)$ de la jornada de la mañana y $245(75,3 \%)$ de la tarde se demoran en conciliar el sueño menos de media hora, lo que es considerado como bueno por este Índice.

El $3^{\circ}$ componente, horas de sueño, los estudiantes de la jornada de la mañana duermen en promedio $6,4 \mathrm{~h}$ y $8,9 \mathrm{~h}$ los de la tarde.
El $4^{\circ}$ componente, eficiencia del sueño, tenemos que en la gran parte de los estudiantes 362 $(97,0 \%)$ de la jornada de la mañana y $325(99,7 \%)$ de la tarde, la eficiencia es mayor o igual a $85 \%$, considerada por este índice como muy bueno.

El $5^{\circ}$ componente, alteraciones de sueño, 167 estudiantes $(44,7 \%)$ de la jornada de la mañana y $89(27,2 \%)$ de la jornada de la tarde, refirieron que jamás despertaron durante la noche. De los que despertaron, las causas en su gran mayoría, 565 adolescentes $(80,9 \%)$ fue para ir al baño.

En el $6^{\circ}$ componente relacionado con el uso de medicamentos para dormir, la gran mayoría no los utiliza, a excepción de 4 estudiantes $(1,1 \%)$ de la jornada de la mañana y 3 de la tarde $(0,9 \%)$.

El $7^{\circ}$ componente, disfunción diurna, los estudiantes de la jornada de la mañana experimentaron una mayor proporción de episodios de som-

Tabla 1. Distribución de los horarios de levantarse de los estudiantes según la jornada de clases de $1^{\circ}$ año (tarde) y $2^{\circ}$ año (mañana)

\begin{tabular}{|lrrrrrr|}
\hline Horarios de despertar & \multicolumn{2}{c}{$\begin{array}{c}\text { Mañana } \\
(\mathbf{n = 3 6 4 )}\end{array}$} & \multicolumn{2}{c}{$\begin{array}{c}\text { Tarde } \\
(\mathbf{n = 3 2 2})\end{array}$} & \multicolumn{2}{c|}{$\begin{array}{c}\text { Total } \\
(\mathbf{n}=\mathbf{6 8 6})\end{array}$} \\
\hline $04: 00-4: 59$ & 4 & $1,1 \%$ & 1 & $0,3 \%$ & 5 & $0,7 \%$ \\
\hline $05: 00-5: 59$ & 97 & $26,6 \%$ & 3 & $0,9 \%$ & 100 & $14,6 \%$ \\
\hline $06: 00-6: 59$ & 248 & $68,1 \%$ & 5 & $1,6 \%$ & 253 & $36,9 \%$ \\
\hline $07: 00-7: 59$ & 15 & $4,1 \%$ & 23 & $7,1 \%$ & 38 & $5,5 \%$ \\
\hline $08: 00-8: 59$ & 0 & $0,0 \%$ & 73 & $22,7 \%$ & 73 & $10,6 \%$ \\
\hline $09: 00-9: 59$ & 0 & $0,0 \%$ & 124 & $38,5 \%$ & 124 & $18,1 \%$ \\
\hline $10: 00-10: 59$ & 0 & $0,0 \%$ & 60 & $18,6 \%$ & 60 & $8,7 \%$ \\
\hline $11: 00$ y más & 0 & $0,0 \%$ & 33 & $10,2 \%$ & 33 & $4,8 \%$ \\
\hline
\end{tabular}

Tabla 2. Distribución de los horarios de ir a dormir de los estudiantes según la jornada de clases de $1^{\circ}$ año (tarde) y $2^{\circ}$ año (mañana)

\begin{tabular}{|lcccccc|}
\hline $\begin{array}{l}\text { Horarios de irse a } \\
\text { dormir }\end{array}$ & \multicolumn{2}{c}{$\begin{array}{c}\text { Mañana } \\
(\mathbf{n = 3 6 4 )}\end{array}$} & \multicolumn{2}{c}{$\begin{array}{c}\text { Tarde } \\
(\mathbf{n = 3 2 2})\end{array}$} & \multicolumn{2}{c|}{$\begin{array}{c}\text { Total } \\
(\mathbf{n = 6 9 9 )}\end{array}$} \\
\hline Antes de 21:00 & 1 & $0,3 \%$ & 0 & $0,0 \%$ & 1 & $0,1 \%$ \\
\hline $21: 00-21: 59$ & 11 & $3,0 \%$ & 2 & $0,6 \%$ & 13 & $1,9 \%$ \\
\hline $22: 00-22: 59$ & 82 & $22,5 \%$ & 23 & $7,1 \%$ & 105 & $15,3 \%$ \\
\hline $23: 00-23: 59$ & 105 & $28,8 \%$ & 66 & $20,5 \%$ & 171 & $24,9 \%$ \\
\hline $00: 00-00: 59$ & 95 & $26,1 \%$ & 111 & $34,5 \%$ & 206 & $30,0 \%$ \\
\hline $01: 00-01: 59$ & 70 & $19,2 \%$ & 120 & $37,3 \%$ & 190 & $27,7 \%$ \\
\hline
\end{tabular}




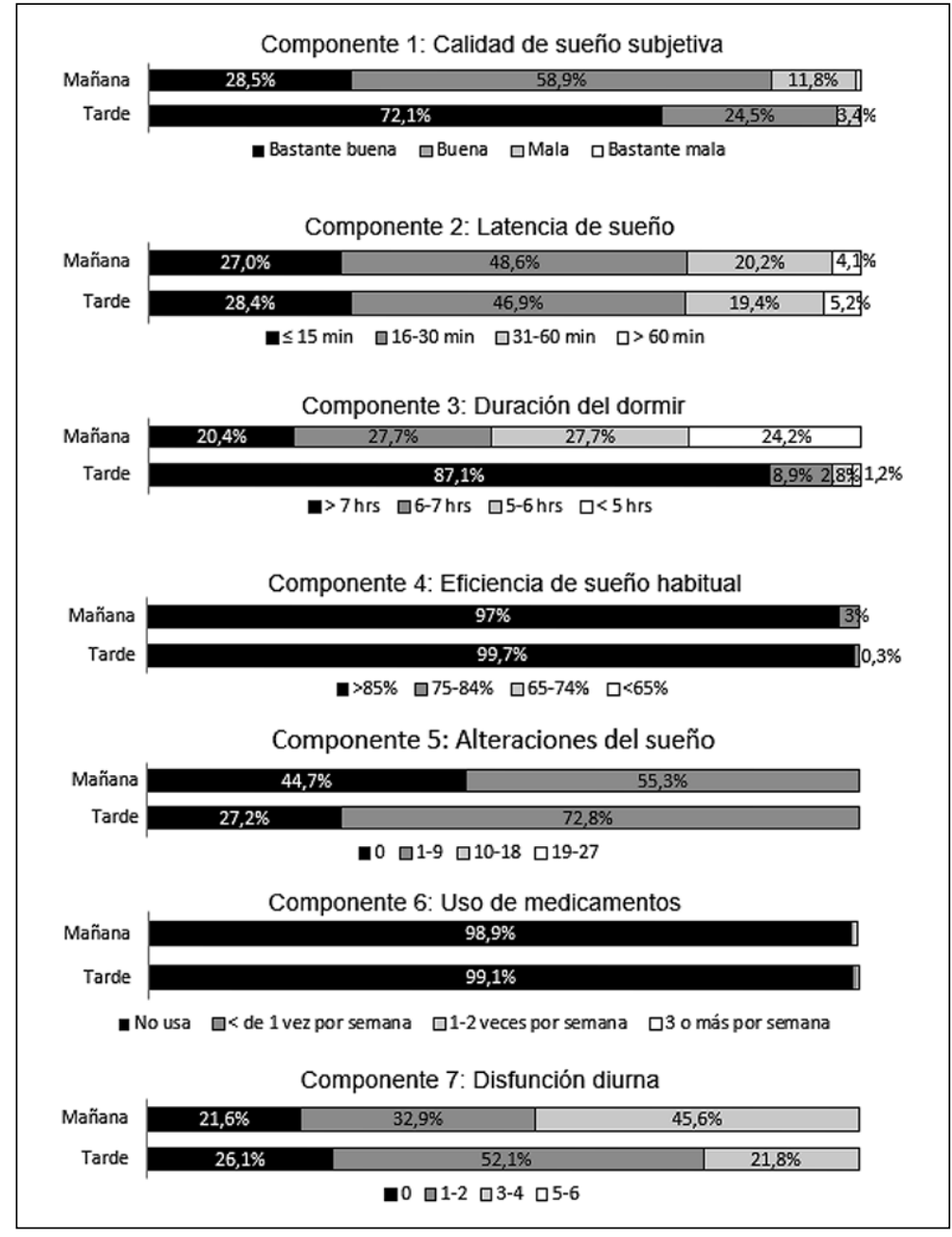

Figura 1. Componentes del Índice de Calidad de Sueño de Pittsburgh - PSQI según jornada escolar de los estudiantes de $1^{\circ}$ (tarde) y $2^{\circ}$ año (mañana) de enseñanza media del Instituto Nacional José Miguel Carrera. nolencia en clases o en el estudio de las materias de 78,5\% (292), que los de la tarde 73,9\% (240).

De la suma de estos componentes obtuvimos la puntuación global de PSQI, lo que corresponde a un promedio total de 4,67 para el total de la muestra, mientras que para los alumnos de la jornada de la mañana fue 5,6, una moda de 7 y un rango de 0-14. Para los alumnos de la tarde, el promedio corresponde a 3,6, la moda 3 y el rango 0-13.

Si consideramos un punto de corte mayor o igual a 5 para la definición de buen o mal dormir (buen dormir $=$ PSQI $<5$; mal dormir $=$ PSQI $\geq 5$ ), encontramos que en la jornada de la mañana la mayoría de los estudiantes, $230(63,2 \%)$ son clasificados como malos dormidores, mientras sólo $30,7 \%$ lo son en la jornada de la la tarde.
Esta diferencia es estadísticamente significativa $(\mathrm{p}<0,01)$ (Figura 2 y 3 ).

Con relación a los promedios de los componentes, podemos ver que la duración del sueño, disfunción diurna, calidad subjetiva y eficiencia del sueño presentan valores significativamente más altos en los estudiantes de la jornada de la mañana, mientras que las alteraciones del sueño son significativamente mayores en la jornada de la tarde. No se aprecian diferencias estadísticamente significativas en el uso de medicamentos ni en la latencia del sueño entre los estudiantes de ambas jornadas (Tabla 3 ).

En relación al rendimiento académico, el promedio de notas del total de asignaturas del primer semestre para los alumnos de la jornada de la ma- 

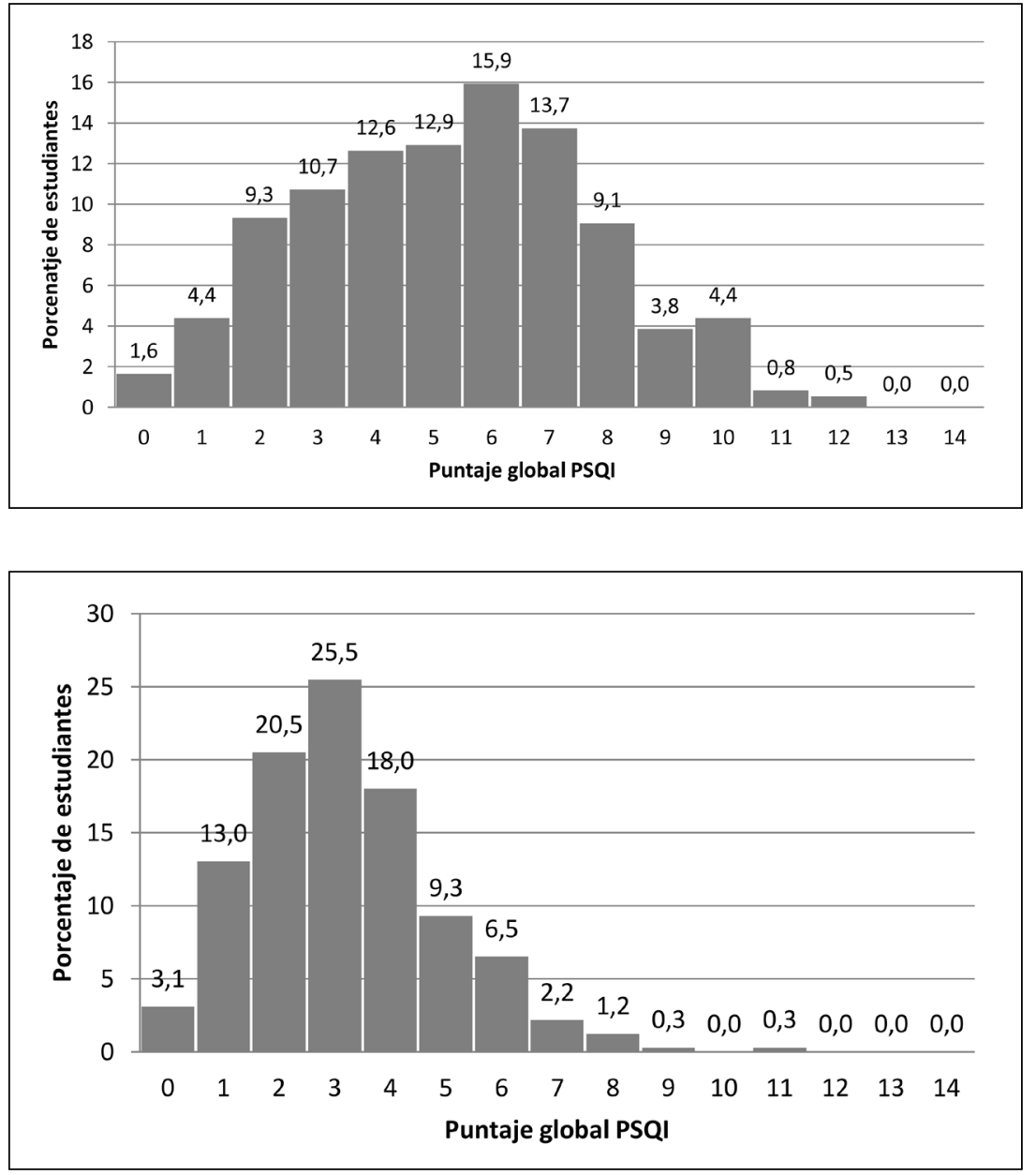

Figura 2. Porcentaje de estudiantes de la jornada de la mañana según puntaje global del Índice de Calidad de Sueño de Pittsburgh - PSQI.
Figura 3. Porcentaje de estudiantes de la jornada de la tarde según puntaje global del Índice de Calidad de Sueño de Pittsburgh - PSQI.
Tabla 3. Promedios de los componentes del Índice de Calidad de Sueño de Pittsburgh-PSQI según jornada de clases

\begin{tabular}{|lcc|}
\hline Componentes & $\begin{array}{c}\text { Mañana } \\
(\mathbf{n}=\mathbf{3 7 3})\end{array}$ & $\begin{array}{c}\text { Tarde } \\
(\mathbf{n}=\mathbf{3 2 6})\end{array}$ \\
\hline Calidad de sueño subjetiva & $0,85^{*}$ & 0,31 \\
\hline Latencia de sueño & 1,02 & 1,01 \\
\hline Duración del sueño & $1,57^{*}$ & 0,18 \\
\hline Eficiencia de sueño & $0,03^{*}$ & 0,00 \\
\hline Alteraciones del sueño & 0,87 & $1,14^{*}$ \\
\hline Uso de medicamentos & 0,02 & 0,01 \\
\hline Disfunción diurna & $1,24^{*}$ & 0,95 \\
\hline Índice calidad de sueño total & $5,6^{*}$ & 3,61 \\
\hline
\end{tabular}

*Diferencia significativa a $p<0,01$. ñana fue 5,9 $(\mathrm{DE}=0,51)$, siendo la nota máxima 7,0 , la mínima 4,8 y la moda 5,8 . Para los alumnos de la jornada de la tarde fue 5,8 $(\mathrm{DE}=0,51)$, siendo la nota máxima 6,8 , la mínima 4,5 y la moda 6,0 (Tabla 4).

$\mathrm{Al}$ revisar la asociación entre los ítems de PSQI y promedio de notas de los estudiantes por jornada, encontramos que en ambos casos la escala se asocia significativa e inversamente con éste. Sin embargo, los ítems de la escala se asocian de forma diferencial según jornada. En la jornada de la mañana, todos los componentes del PSQI, salvo eficiencia del sueño, están asociados inversa y significativamente con rendimiento, mientras que en el caso de la tarde esto sólo ocurre con los componentes de latencia y alteraciones del sueño (Tablas 5 y 6 ). 
Finalmente, se utilizó la técnica de regresión lineal con el fin de determinar los principales factores asociados a los puntajes del PSQI. Para esto, se utilizó como variable dependiente el

Tabla 4. Notas promedio del primer semestre 2016 según jornada

\begin{tabular}{|lcccc|}
\hline Notas & \multicolumn{2}{c}{ Mañana } & \multicolumn{2}{c|}{ Tarde } \\
& n & \% & n & \% \\
\hline 7,0 a 6,0 & 182 & 50 & 142 & 44,1 \\
\hline 5,9 a 5,0 & 169 & 46,4 & 158 & 49,1 \\
\hline 4,9 a 4,0 & 12 & 3,6 & 22 & 6,8 \\
\hline n total & 364 & 100 & 322 & 100 \\
\hline
\end{tabular}

PSQI. Como variables independientes se utilizó la jornada escolar, así como una recodificación de las horas de despertar $(1=$ antes de las $6 \mathrm{AM}$; $0=$ después de las $6 \mathrm{AM})$ y dormir $(1=$ Después de las $12 \mathrm{AM} ; 0=$ antes de las $12 \mathrm{AM})$. Los resultados del modelo final muestran un ajuste razonable, $(\mathrm{R} 2=0,322)$. Así, observamos que los principales predictores del PSQI están asociados a los horarios de dormir, mostrando efectos en aquellos estudiantes que van a dormir después de las $12 \mathrm{AM}$ $(\beta=0,394, p<0,001)$ y en aquellos que despiertan antes de las $6 \mathrm{AM}(\beta=0,139, \mathrm{p}<0,001)$. Por otro lado, la jornada escolar también está asociada a los puntajes del PSQI, mostrando que asistir en la jornada de la tarde predice un menor puntaje del PSQI $(\beta=-0,123, \mathrm{p}<0,001)$ (Tabla 7).

Tabla 5. Correlación entre componentes del Índice de Calidad de Sueño de Pittsburgh-PSQI y promedio de notas semestrales - Estudiantes mañana

\begin{tabular}{|c|c|c|c|c|c|c|c|c|c|}
\hline & $\underset{1}{\text { Comp }}$ & $\underset{2}{\text { Comp }}$ & $\begin{array}{c}\text { Comp } \\
3\end{array}$ & $\begin{array}{c}\text { Comp } \\
4\end{array}$ & $\begin{array}{c}\text { Comp } \\
5\end{array}$ & $\begin{array}{c}\text { Comp } \\
6\end{array}$ & $\underset{7}{\text { Comp }}$ & PSQI & $\begin{array}{c}\text { Pro- } \\
\text { medio }\end{array}$ \\
\hline Calidad de sueño subjetiva & 1 & & & & & & & & \\
\hline Latencia de sueño & $0,213^{* *}$ & 1 & & & & & & & \\
\hline Duración del sueño & $0,682^{* *}$ & $0,115^{*}$ & 1 & & & & & & \\
\hline Uso de medicamentos & $0,239^{* *}$ & $0,378^{* *}$ & $0,237^{* *}$ & 1 & & & & & \\
\hline Alteraciones del sueño & $0,106^{*}$ & $0,251^{* *}$ & 0,081 & 0,042 & 1 & & & & \\
\hline Eficiencia de sueño & 0,022 & 0,029 & $0,133^{*}$ & $-0,018$ & $-0,026$ & 1 & & & \\
\hline Disfunción diurna & $0,292^{* *}$ & $0,178^{* *}$ & $0,223^{* *}$ & 0,048 & $0,240^{* *}$ & 0,033 & 1 & & \\
\hline PSQI & $0,707^{* *}$ & $0,554^{* *}$ & $0,708^{* *}$ & $0,352^{* *}$ & $0,543^{* *}$ & $0,147^{* *}$ & $0,587^{\star *}$ & 1 & \\
\hline Promedio & $-0,168^{* *}$ & $-0,175^{* *}$ & $-0,107^{\star}$ & $-0,111^{*}$ & $-0,136^{* *}$ & $-0,031$ & $-0,125^{*}$ & $-0,226^{* *}$ & 1 \\
\hline
\end{tabular}

*La correlación es significativa en el nivel 0,05 (bilateral). ** La correlación es significativa en el nivel 0,01 (bilateral).

Tabla 6. Correlación entre componentes del Índice de Calidad de Sueño de Pittsburgh-PSQI y promedio de notas semestrales - Estudiantes tarde

\begin{tabular}{|c|c|c|c|c|c|c|c|c|c|}
\hline & $\underset{1}{\text { Comp }}$ & $\underset{2}{\text { Comp }}$ & $\begin{array}{c}\text { Comp } \\
3\end{array}$ & $\begin{array}{c}\text { Comp } \\
4\end{array}$ & $\underset{5}{\text { Comp }}$ & $\underset{6}{\text { Comp }}$ & $\underset{7}{\text { Comp }}$ & PSQI & $\begin{array}{c}\text { Pro- } \\
\text { medio }\end{array}$ \\
\hline Calidad de sueño subjetiva & 1 & & & & & & & & \\
\hline Latencia de sueño & $0,141^{*}$ & 1 & & & & & & & \\
\hline Duración del sueño & $0,365^{* *}$ & 0,051 & 1 & & & & & & \\
\hline Uso de medicamentos & $0,179^{* *}$ & $0,134^{*}$ & $0,297^{\star *}$ & 1 & & & & & \\
\hline Alteraciones del sueño & 0,063 & $0,297^{* *}$ & 0,062 & $0,114^{*}$ & 1 & & & & \\
\hline Eficiencia de sueño & $-0,047$ & 0,046 & $-0,027$ & $-0,004$ & 0,031 & 1 & & & \\
\hline Disfunción diurna & $0,159^{* *}$ & $0,298^{* *}$ & 0,108 & 0,004 & $0,232^{* *}$ & 0,005 & 1 & & \\
\hline PSQI & $0,482^{* *}$ & $0,677^{* *}$ & $0,434^{* \star}$ & $0,250^{* *}$ & $0,665^{* *}$ & 0,052 & $0,617^{\star \star}$ & 1 & \\
\hline Promedio & $-0,036$ & $-0,163^{* *}$ & 0,072 & 0,021 & $-0,203^{* *}$ & 0,029 & $-0,069$ & $-0,165^{* *}$ & 1 \\
\hline
\end{tabular}

*La correlación es significativa en el nivel 0,05 (bilateral). ** La correlación es significativa en el nivel 0,01 (bilateral). 
Tabla 7. Coeficientes de regresión logística. Variable dependiente: mal dormir

\begin{tabular}{|lccccc|}
\hline & \multicolumn{2}{c}{$\begin{array}{c}\text { Coeficientes } \\
\text { no estandarizados } \\
\text { B }\end{array}$} & $\begin{array}{c}\text { Coeficientes } \\
\text { Estandarizados } \\
\text { Betán }\end{array}$ & t & Sig. \\
\hline (Constante) & 6,633 & 0,294 & & 22,553 & 0,000 \\
\hline Jornada & $-2,218$ & 0,185 & $-0,420$ & $-11,983$ & 0,000 \\
\hline Vive en Santiago Centro & $-1,433$ & 0,371 & $-0,123$ & $-3,864$ & 0,000 \\
\hline Despertar antes de las 6 AM & 1,014 & 0,249 & 0,139 & 4,078 & 0,000 \\
\hline Dormir después de las12 AM & 2,105 & 0,175 & 0,394 & 12,009 & 0,000 \\
\hline
\end{tabular}

\section{Discusión}

La etapa de la adolescencia es una fase de transición bio-psico-social, la cual influye en los patrones de sueño ${ }^{11}$. Según la Academia Americana de Medicina del Sueño (AASM) en la Conferencia de Consenso (2016) sobre el tiempo de duración del sueño, estableció la recomendación que los adolescentes sanos de 13 a 18 años de edad deben dormir de 8 a 10 h por día para promover una salud óptima ${ }^{12,13}$. Así, tenemos que la hora de ir a dormir de la mayoría de nuestros estudiantes de la jornada de la mañana, es entre las 23:0001:00 h y los de la tarde entre las 00:00-02:00 h. Estos horarios concuerdan con una investigación realizada por Fontana y cols., en la cual evidencia que la mayoría de su población de estudiantes se acuesta entre las 23:00-2:00 de la mañana ${ }^{14}$. Estas tardanzas nocturnas, según McGlinchey y col, son frecuentes entre los adolescentes con estimaciones de más de $25 \%$ que se van a dormir después de la medianoche ${ }^{15}$. Lo que es importante según Merikanto y cols, al relacionarlo con el rendimiento académico, quienes realizaron un estudio epidemiológico con una muestra de 384.076 estudiantes en Finlandia entre los cuales se incluía alumnos de grados escolares equivalentes a $1^{\circ}$ y $2^{\circ}$ año de enseñanza media de nuestro país, concluyendo que cuanto más tarde la hora de acostarse de los adolescentes, menor será su rendimiento escolar ${ }^{16}$. Contrariamente a lo anterior, Quebedo-Blasco y col, refiere que la hora de acostarse y la hora de levantarse, no influyen significativamente sobre el rendimiento escolar ${ }^{17}$.

Para cumplir con los deberes escolares, los alumnos de la jornada de la mañana se levantan en promedio 3 h más temprano que los de la tarde, lo que hace una gran diferencia en las horas de sueño entre ambas jornadas escolares. Estos resultados están en línea con la conclusión de Morgenthaler 2016, quien refiere que los tiempos de inicio más tardíos en la mañana de las clases están asociados con el aumento de la duración total del sueño ${ }^{18}$. Así, los estudiantes de la jornada de la mañana duermen en promedio $6,4 \mathrm{~h}$ y los de la tarde $8,9 \mathrm{~h}$, siendo este componente, duración del sueño, el que más aumentó nuestra escala PSQI y el que hizo la diferencia entre ambas jornadas. Lo que es relevante, ya que varios autores encontraron que sus estudios proporcionaban pruebas adicionales sobre que el aumento de las horas de sueño se asocia con un mejoramiento de las calificaciones ${ }^{13,17,19}$. Contrariamente a lo anterior, Eliasson y cols, en sus estudios encontraron que no hay una correlación significativa entre el tiempo total de sueño y el rendimiento académico de los estudiantes ${ }^{20}$. De esta manera, para verificar las opuestas afirmaciones anteriores, Wheaton y cols, realizaron un meta-análisis de 38 artículos, comprobando que el sueño insuficiente en los adolescentes está asociado con las calificaciones académicas más bajas. Sin embargo, también encontró que los estudiantes que duermen más tiempo también reportaron menos estudios y más bajos puntajes en el examen de admisión a las universidades en Estados Unidos ${ }^{21}$.

Nuestros estudiantes de ambas jornadas presentan un buen rendimiento académico que se asocia con la escala PSQI pero la gran diferencia está en que los estudiantes de la jornada de la mañana duermen menos horas. Esto puede tener relación con las conclusiones de Fuligni y cols, quienes refieren que reducir el sueño por el desempeño académico puede resultar en una mayor 
disminución en la salud, que en una disminución del rendimiento académico ${ }^{11}$. En la misma línea Belenky y cols, realizaron una investigación sobre el rendimiento de las labores diarias y la restricción del sueño, sus resultados sugieren que el cerebro se adapta a una restricción crónica leve o moderada del sueño, la cual es suficiente para estabilizar el rendimiento, aunque a un nivel reducido ${ }^{22}$.

Un hallazgo interesante en nuestro estudio es que el promedio del índice PSQI de los estudiantes de la jornada de la mañana puntuaron como malos dormidores $(5,57)$, mientras que ellos consideraban que su calidad de sueño era buena o muy buena (en total $87,4 \%$ de los estudiantes), lo que sugiere una discordancia entre la calificación subjetiva que el encuestado adjudica a su dormir y el puntaje global de la calidad de sueño, esto mostraría una sobreestimación de la calidad subjetiva del sueño por parte de los estudiantes, siendo este resultado muy similar al obtenido por otros autores como Sierra y cols y Rosales y cols, ${ }^{23,24}$. Una plausible explicación, según estos autores sería el tener creencias disfuncionales acerca del sueño, conocimiento insuficiente o pocas prácticas de hábitos saludables de sueño ${ }^{23,24}$.

\section{Referencias}

1. Kloss JD, Nash CO, Horsey SE, Taylor DJ. The delivery of behavioral sleep medicine to college students. J Adolesc Health 2011; 48 (6): 553-61.

2. Bartel K, Williamson P, van Maanen A, Cassoff J, Meijer $\mathrm{AM}$, Oort F, et al. Protective and risk factors associated with adolescent sleep: findings from Australia, Canada, and The Netherlands. Sleep Med 2016; 26: 97-103.

3. Gustafsson ML, Laaksonen C, Aromaa M, Asanti R, Heinonen OJ, Koski P et al. Association between amount of sleep, daytime sleepiness and health-related quality of life in schoolchildren. J Adv Nurs 2016; 72 (6): 1263-72.

4. Buysse DJ, Reynolds CF 3rd, Monk TH, Berman SR, Kupfer DJ. The Pittsburgh Sleep Quality Index: a new instrument for psychiatric practice and research. Psychiatry Res 1989; 28 (2): 193-213.

5. Jiménez-Genchi A, Monteverde-Maldonado E, Nenclares-Portocarrrero A, Esquivel-Adame G, De la Vega-Pacheco A. Confiabilidad y análisis factorial de la versión en español del índice de calidad de sueño de Pittsburgh en pacientes psiquiátricos. Gac Méd Méx 2008; 144 (6): 491-6.

6. Ministerio de Salud. Encuesta Nacional de Salud ENS
2009-2010 [Internet]. Santiago, Chile: Ministerio de Salud; 2010. Tomo I [citado el 17 de octubre de 2016]. Disponible en: http://web.minsal.cl/portal/url/item/ bcb03d7bc28b64dfe040010165012d23.pdf

7. Instituto Nacional José Miguel Carrera. El Instituto: Reseña histórica [interrnet]. Santiago, Chile [citado el 12 de octubre de 2016]. Disponible en: https://institutonacional.cl/el-instituto/resena-historica/.

8. Royuela A, Macías JA. Propiedades clinimétricas de la versión castellana del Cuestionario de Pittsburgh. Vigilia- Sueño 1997; 9 (2): 81-94.

9. Ministerio de Educación Pública. Fija el texto definitivo del reglamento de calificaciones, exámenes y promociones de alumnos de educación secundaria. Decreto 1.480. Vistos el oficio $\mathrm{N}^{\circ} 34$, de la Superintendencia de Educación Pública, y el oficio $N^{\circ}$ 537. de la Dirección de Educación Secundaria. TITULO II De las Calificaciones de Rendimiento. Artículo 10. Santiago, Chile. 16 de marzo de 1961. Disponible en: https://www.leychile.cl/ Navegar?idNorma=1044541

10. Ministerio de Educación Pública. Decreto 112 exento. Establece disposiciones para que los establecimientos educacionales elaboren su reglamento de evaluación y reglamenta la promoción de alumnos de 1er y 2 do año de enseñanza media. Ambas modalidades. Santiago, Chile. 20 de abril de 1999. Disponible en: https://www. leychile.cl/Navegar?idNorma $=135826$

11. Fuligni AJ, Arruda EH, Krull JL, Gonzales NA. Adolescent sleep duration, variability, and peak levels of achievement and mental health. Child Dev 2017; Jan 27. Disponible en: http://onlinelibrary.wiley.com/ doi/10.1111/cdev.12729/epdf

12. Paruthi S, Brooks LJ, D’Ambrosio C, Hall WA, Kotagal S, Lloyd RM, et al. Recommended amount of sleep for pediatric populations: a consensus statement of the American Academy of Sleep Medicine. J Clin Sleep Med 2016; 12 (6): 785-86.

13. Paruthi S, Brooks LJ, D’Ambrosio C, Hall WA, Kotagal S, Lloyd RM, et al. Consensus statement of the American Academy of Sleep Medicine on the recommended amount of sleep for healthy children: methodology and discussion. J Clin Sleep Med 2016; 12 (11): 1549-61.

14. Fontana S, Raimondi W, Rizzo M. Calidad de sueño y atención selectiva en estudiantes universitarios: estudio descriptivo transversal. Medwave 2014; 14 (8). Disponible en: https://www.medwave.cl/link.cgi/Medwave/ Estudios/Investigacion/6015.

15. McGlinchey E, Harvey A. Risk behaviors and negative health outcomes for adolescents with late bedtimes. J Youth Adolesc 2015; 44 (2): 478-88.

16. Merikanto I, Lahti T, Puusniekka R, Partonen T. Late 
bedtimes weaken school performance and predispose adolescents to health hazards. Sleep Med 2013; 14 (11): 1105-11.

17. Quevedo-Blasco V, Quevedo-Blasco R. Influencia del grado de somnolencia, cantidad y calidad de sueño sobre el rendimiento académico en adolescentes. Int J Clin Health Psychol 2011; 11 (1): 49-65.

18. Morgenthaler TI, Hashmi S, Croft JB, Dort L, Heald JL, Mullington J. High school start times and the impact on high school students: what we know, and what we hope to learn. J Clin Sleep Med 2016; 12 (12): 1681-9.

19. Cole JS. Do later wake times and increased sleep duration of 12th graders result in more studying, higher grades, and improved SAT/ACT test scores?. Sleep Breath 2016; 20 (3): 1053-7.

20. Eliasson A, Eliasson A, King J, Gould B, Eliasson A. Association of sleep and academic performance. Sleep
Breath 2002; 6 (1): 45-8.

21. Wheaton AG, Chapman DP, Croft JB. School Start Times, Sleep, Behavioral, Health, and Academic Outcomes: A Review of the Literature. J Sch Health 2016; 86 (5): 363-81.

22. Belenky G, Wesensten NJ, Thorne DR, Thomas ML, Sing HC, Redmond DP, et al. Patterns of performance degradation and restoration during sleep restriction and subsequent recovery: a sleep dose-response study. J Sleep Res 2003; 12 (1): 1-2.

23. Sierra JC, Jiménez-Navarro C, Martín-Ortíz JD. Calidad del sueño en estudiantes universitarios: importancia de la higiene del sueño. Salud Mental 2002; 25: 35-43.

24. Rosales E, Egoavil M, La Cruz C, Rey de Castro J. Somnolencia y calidad del sueño en estudiantes de medicina de una universidad peruana. An Fac Med Lima 2007; 68(2): $150-8$ 